\title{
Demand And Equilibrium In A Network Of Oligopolistic Markets
}

Natalia Kalashnikova, University of Nuevo Leon, Monterrey, Mexico

Vyacheslav Kalashnikov, Monterrey Institute of Technologies, Monterrey, Mexico

\begin{abstract}
In this paper, we consider an oligopolistic model of electricity market with conjectures concerning the price variations depending upon the agents' production increase or increase. By introducing the notion of an exterior equilibrium and a special type of a producer's cost function, we establish the existence and uniqueness of the exterior (conjectured) equilibrium. Then we introduce the notion of an interior stable equilibrium and analyze the behavior of groups of consumers with different consumption abilities. The proposed techniques allow us to make a qualitative description of dependence of the market price upon the active demand component.
\end{abstract}

\section{Introduction}

W

hen studying the oligopolistic market in the framework of the classical models [1] - [3], besides the questions of an equilibrium existence and its computation, a lot of attention is usually paid to comparing the latter with the equilibrium in the perfect competition model. Recently [4] - [5], both models were included in a united class of oligopoly models where the degree of influence on the whole situation by each agent is modeled by some special parameters (influence coefficients). If we set these influence coefficients in an exogenous way, then the demand structure does not affect the production regime, i.e. the dependence of output values upon the market price. Although a deformation of the demand curve leads to a variation of equilibrium, the possible modeling of the deformation influence in such models is limited. For example, it is difficult to trace a rapid (or even jump-like) variation of the market price, a stable price under the demand growth (within certain limits), and a series of other phenomena.

However, if one does not consider the influence coefficients as given beforehand but includes them in the definition of the equilibrium itself, together with the equilibrium price and production volumes, one obtains a possibility to simulate the above-mentioned effects. Such a notion of equilibrium was introduced in the paper by V.A. Bulavsky [6]. There, the influence coefficients are determined by a special verification procedure that checks their consistency. The procedure in [6] supposes that each agent can observe the variations of the aggregate production output as a response to his own output variations and thus estimate his influence. The choice of the aggregate production volume as the observed parameter was done due to the fact that the classical Cournot model was taken as the basis of the developed model. Nevertheless, one can assume that it is more natural to observe the market price instead of the aggregate output, and hence estimate each agent's influence upon the price. Such an approach is developed in papers [7] and [9], which allowed to relax the conditions imposed on the demand function and keep on in essence only one of them, namely its being non-increasing when the price increases. A technical condition for the latter function of being piecewise smooth is also kept valid.

In such a framework in [7] and [9], the equilibrium existence was established, and certain consequences of the demand structure variations were examined. Namely, two types of variations were illustrated. The first one is related to the fall down of consumption ability of one group of consumers with the consumption ability of another group of consumers kept intact. It turned out that the proposed model predicted three stages of the process: (i) the price going down with the aggregate production output kept constant, (ii) the price going down further with the simultaneous production decreasing and a deficit arising, (iii) and at last, a sharp jump of the price without production growth, and the deficit disappearing on account of the poorer consumers abandoning the market. 
Before mentioning the second type of the demand structure variations we insert a certain elucidation. In the classical oligopoly models, the aggregate bargain volume decreases as the price grows up. This fact goes in line with the postulate of the price falling down as the supply increases. However, there exists a dual (in a certain sense) postulate: the price increases as the demand goes up. In order to remove this seeming contradiction, one should make distinction between two kinds of demand: a passive and an active one. The standard demand function in the classical oligopoly models describes the passive demand: consumers are waiting for a good to be proposed to them at some price and then decide to buy it or not to buy. However, the active demand does not depend on the price and constitutes the additional component of the aggregate demand. So when the active demand increases the market price should grow up. It is the growth of the active demand component that is the second type of the demand structure variations examined in [7] and [9]. The active component may reflect both an agitating demand and the demand related to some needs outside the model. For example, it could be a demand arising because of some military actions, development of the defense material production, etc. It was shown in [7] and [9] that the active demand growing, in the presence of a group of passive consumers, also provides three phases to be observed: (i) the price kept invariable while the production goes up, (ii) the constant price with the constant production volume and hence, a deficit appearing, (iii) and at last, the price jumping up as the market is abandoned by the passive consumers, and only the active demand is satisfied thereafter.

An interesting phenomenon was also mentioned in [7] and [9]: with both the first and the second type of the demand structure variations, the process is not invertible completely. For example, if the leap in price is followed by the active demand going down, then the price does not return to the initial level unless the active demand becomes lower than the initial one! Similarly, in the second example, the initial price is not reached unless the poorer group is drawn up to the state of the richer group but not to the poor initial state.

In the paper, we apply the mathematical model from [7], [9] to an electricity market in which every firm producing the electricity may have several generating units. Therefore, their total production cost function is formed by minimizing the total production cost. The model is specified in the next section. We prove the existence of exterior equilibrium and illustrate that by simple examples. Then by approximating thus obtained piecewise linear total production cost function by a special quadratic function, we are able to introduce the notion of interior equilibrium and realize the qualitative analysis of the interactions between the active demand and the equilibrium values.

\section{Model Specification}

Consider $n \geq \mathbf{3}$ firms-producers of the electric power numbered by $\boldsymbol{i}=\mathbf{1}, \ldots, \boldsymbol{n}$, and $\boldsymbol{j}=\mathbf{1}, \mathbf{2}, \ldots, \boldsymbol{n}_{\boldsymbol{i}}$ denote the generating units owned by firm $i$. We also introduce the following exogenous variables, decision variables, and profits, similar to those in [8]:

Exogenous variables:

$\boldsymbol{c}_{i j}$

$$
\begin{aligned}
& \text { CAP }_{i j} \\
& \text { LOSS } \\
& \text { RES_RATIO }_{i j} \\
& \text { FOR }_{i j}
\end{aligned}
$$

\section{Decision variables:}

$x_{i j}$

$x r_{i j}$ constant marginal cost of unit $\boldsymbol{j}$ owned by firm $\boldsymbol{i}$

capacity of unit $\boldsymbol{j}$ owned by firm $\boldsymbol{i}$

percentage loss incurred over transmission and distribution lines required reserve ratio for unit $\boldsymbol{j}$ owned by firm $\boldsymbol{i}$

forced outage rate for unit $j$ owned by firm $i$

megawatts (MW) of electricity generated by unit $j$ owned by firm $\boldsymbol{i}$

MW of capacity required for reserves by unit $\boldsymbol{j}$ owned by firm $\boldsymbol{i}$

Consumers demand is described by a demand function $\boldsymbol{G}(\boldsymbol{p})$, whose argument $\boldsymbol{p}$ is the price proposed by the producers. An active demand $\boldsymbol{D}$ is non-negative and does not depend upon the price. The demand function is 
assumed to be non-negative and non-increasing, and also continuously differentiable everywhere except for a finite number of points in which both the function $G(p)$ and its derivative $G^{\prime}(p)$ may be discontinuous. Because of that, we denote by $\boldsymbol{G}(\boldsymbol{p})$ the demand function's left limit at the point $\boldsymbol{p}$, whereas the right limit is denoted by $\boldsymbol{g}(\boldsymbol{p})$. It is straightforward that $G(p)=g(p)$ at the continuity points while $G(p)>g(p)$ at the points of discontinuity. In the latter case, $\boldsymbol{G}(\boldsymbol{p})$ can be treated as the potential market capacity, and $\boldsymbol{g}(\boldsymbol{p})$-- as the lower limit of supply preventing the price increase. To describe the model, we introduce the followings groups of constraints.

\section{Demand constraints}

The equilibrium between the demand and supply for a given price value $\boldsymbol{p}$ is stipulated by the following pair of inequalities

$g(p)+D \leq \sum_{i=1}^{n} \sum_{j=1}^{n_{i}} x_{i j}(1-L O S S) \leq G(p)+D$

Reserve constraints

$$
\sum_{j=1}^{n_{i}}\left(x r_{i j}-R E S_{-} R A T I O_{i j} x_{i j}\right) \geq 0 \quad \forall i=1,2, \ldots, n .
$$

\section{Capacity constraints}

$x_{i j}+x r_{i j}=C A P_{i j}\left(1-F O R_{i j}\right) \quad \forall i=1, \ldots, n ; \forall j=1, \ldots, n_{i}$.

Non-negativity constraints

$$
x_{i j} \geq \mathbf{0}, \quad x r_{i j} \geq 0, \quad \forall i, j
$$

That is, inequalities (1) model the generation requires to meet demand by adjusting the amount of electricity produced by each generating unit by a loss factor $\mathbf{L O S S}$.

The reserve ratio constraints, inequalities (2), require each firm to maintain a spinning reserve to satisfy regional reliability requirements. Every firm that generates electricity is required to preserve capacity up to a set percentage of electricity it generates $\left(\boldsymbol{R E S} \_\boldsymbol{R A T I O _ { i j }}\right)$.

The generation capacity constraints, equations (3), ensure that the sum of the electricity produced by each unit $\boldsymbol{x}_{i j}$ and the capacity used as spinning reserve $\boldsymbol{x r}_{i j}$ does not exceed the adjusted total capacity $\boldsymbol{C A} \boldsymbol{P}_{i j}$ of the unit. To account for unscheduled maintenance and outages, $\boldsymbol{C A P}_{i j}$ is adjusted to expected capacity by using the forced outage rate $\boldsymbol{F O R}_{i j}$ specific to that unit. The $\boldsymbol{F O R}_{i j}$ represents the probability of an unplanned outage in any given hour for that unit.

Now we denote by $\boldsymbol{q}_{i}$ the total output of electricity by firm $\boldsymbol{i}$, that is,

$q_{i}=\sum_{j=1}^{n_{i}} x_{i j}, \quad i=1,2, \ldots, n$,

and introduce the cost functions $f_{i}\left(\boldsymbol{q}_{i}\right), \boldsymbol{i}=\mathbf{1}, \mathbf{2}, \ldots, \boldsymbol{n}$, as follows: 


$$
f_{i}\left(q_{i}\right)=\min \left\{\sum_{j=1}^{n_{i}} c_{i j} x_{i j} \mid \text { subject to (2), (3), (4) and (5) }\right\} .
$$

The following result is a direct consequence of the Linear Programming Sensitivity Analysis.

Lemma 1. Each function $f_{i}\left(\boldsymbol{q}_{i}\right)$ defined by (6) is piece-wise linear and convex.

The latter implies that at each point $\boldsymbol{q}_{i}$ where the value $\boldsymbol{f}_{\boldsymbol{i}}\left(\boldsymbol{q}_{i}\right)$ is finite, i.e. $\boldsymbol{f}_{\boldsymbol{i}}\left(\boldsymbol{q}_{i}\right)<+\infty$, the cost function's subdifferential is non-empty, i.e.

$$
\partial f_{i}\left(q_{i}\right) \equiv\left\{s \in R \mid f_{i}\left(q_{i}+\eta\right) \geq f_{i}\left(q_{i}\right)+s \cdot \eta, \quad \forall \eta \in R\right\} \neq \varnothing .
$$

At the points of differentiability, $\partial f_{i}\left(q_{i}\right)=\left\{f^{\prime}{ }_{i}\left(q_{i}\right)\right\}$.

Example 1. Let $n=1, n_{1}=3, C_{A P}\left(1-F O R_{1 j}\right)=7, j=1,2,3 ;, c_{11}=2, c_{12}=1, c_{13}=1 \quad$ and $R E S_{-} R A T I O_{11}=1 / 4, R E S S_{-} R A T I O_{12}=1 / 8, R E S_{-} R A T I O_{13}=1 / 2$. Then it is easy to verify that

$$
f_{1}\left(q_{1}\right) \equiv f(q)=\min 2 x_{1}+x_{2}+x_{3}
$$

subject to

$$
\begin{gathered}
5 / 4 x_{1}+9 / 8 x_{2}+3 / 2 x_{3} \leq 21, \\
x_{1}+x_{2}+x_{3}=q, \\
0 \leq x_{j} \leq 7, j=1,2,3 .
\end{gathered}
$$

Solving this linear programming problem, we find that

Therefore,

$$
f(q)= \begin{cases}q, & 0 \leq q \leq 14 \\ 2 q-14, & 14<q \leq 16.1 \\ 7 q-94.5, & 16.1<q \leq 16 \frac{11}{12} \approx 16.917 \\ +\infty, & q>16.917\end{cases}
$$

$$
\partial f(q)= \begin{cases}\{1\}, & 0 \leq q<14 \\ {[1,2],} & q=14 \\ \{2\}, & 14<q<16.1 \\ {[2,7],} & 16.1<q<16 \frac{11}{12} \approx 16.917 \\ \varnothing, & q \geq 16.917\end{cases}
$$

Now we specify a notion of equilibrium. Producer $\boldsymbol{i}$ chooses its output volume $\boldsymbol{q}_{\boldsymbol{i}}$ so as to maximize its profit value $\boldsymbol{p} \cdot \boldsymbol{q}_{i}-\boldsymbol{f}_{\boldsymbol{i}}\left(\boldsymbol{q}_{i}\right), \boldsymbol{q}_{i} \geq \mathbf{0}$ assuming that the choice may affect the price value $\boldsymbol{p}$. The latter assumption could be defined by a conjectured dependence of the price $\boldsymbol{p}$ upon the output value $\boldsymbol{q}_{i}$. If so, the first order maximum condition to describe the equilibrium would have the form

$\left.\begin{array}{l}\text { If } \boldsymbol{q}_{i}>\mathbf{0}, \text { then } 0 \in \\ \left.\text { If } \boldsymbol{q}_{i}=\mathbf{0}, \text { then } 0 \geq\right\}\end{array}\right\} p+q_{i} \frac{\partial p}{\partial q_{i}}-\partial f_{i}\left(q_{i}\right)$ 
Thus we see that to describe the agent's behavior, not the dependence of $\boldsymbol{p}$ upon $\boldsymbol{q}_{i}$ itself but its derivative $\frac{\partial \boldsymbol{p}}{\partial \boldsymbol{q}_{i}}=-\boldsymbol{v}_{\boldsymbol{i}}$ is important. We introduce the minus here in order to deal with the positive values of $\boldsymbol{v}_{\boldsymbol{i}}$. Of course, the conjectured dependence of $\boldsymbol{p}$ upon $\boldsymbol{q}_{i}$ must provide (at least local) concavity of the $\boldsymbol{i}$-th agent's conjectured profit as the function of his output. Otherwise one cannot guarantee the profit to be maximized (but not minimized). As we prove that the cost functions $\boldsymbol{f}_{\boldsymbol{i}}\left(\boldsymbol{q}_{i}\right)$ are convex, then the concavity of the product $\boldsymbol{p} \cdot \boldsymbol{q}_{i}$ will do. For instance, it is enough to assume the coefficient $\boldsymbol{v}_{\boldsymbol{i}}$ (called from now on as the $\boldsymbol{i}$-th agent's influence coefficient) to be non-negative and constant. Then the conjectured local dependence of the profit upon the production output $\eta_{i}$ has the form $\left[p-v_{i}\left(\eta_{i}-q_{i}\right)\right] \eta_{i}-f_{i}\left(\eta_{i}\right)$, while the maximum condition at $\eta_{i}=q_{i}$ is provided by the relations

$$
\begin{cases}p-v_{i} q_{i} \in \partial f_{i}\left(q_{i}\right), & q_{i}>0 \\ p \leq f_{i}^{\prime}(0), & q_{i}=0\end{cases}
$$

In a sense, condition (9) determines an optimal response of firm $\boldsymbol{i}$ to a given price $\boldsymbol{p}$.

Example 2. In continuation of Example 1, assume that $\boldsymbol{v}_{\mathbf{1}} \equiv \boldsymbol{v}=\mathbf{0 . 5}$. Now we can construct the optimal response curve by producer 1 , namely $\boldsymbol{q}_{\mathbf{1}} \equiv \boldsymbol{q} \equiv \boldsymbol{q}(\boldsymbol{p}, \boldsymbol{v})$, whose graph at a point $\boldsymbol{p}$ is the set of values $\boldsymbol{q}_{\mathbf{1}} \equiv \boldsymbol{q}$ satisfying (9). As condition (9) in our particular case is reduced to

$$
\begin{cases}p \leq 1, & q=0 ; \\ p-0.5 q=1, & 0 \leq q<14 ; \\ p-0.5 q \in[1,2], & q=14 ; \\ p-0.5 q=2, & 14<q<16.1 \\ p-0.5 q \in[2,7], & q=16.1 ; \\ p-0.5 q=7, & 16.1<q \leq 16 \frac{11}{12} \approx 16.917\end{cases}
$$

It is not difficult to verify that we thus obtain the following piece-wise linear function:

$$
q(p, 0.5)= \begin{cases}0, & 0 \leq p \leq 1 ; \\ 2(p-1), & 1<p \leq 8 ; \\ 14, & 8<p \leq 9 ; \\ 2(p-2), & 9<p \leq 10.05 ; \\ 16.1, & 10.05<p \leq 15.05 ; \\ 2(p-7), & 15.05<p \leq 15 \frac{11}{24} \approx 15.458\end{cases}
$$

Lemma 2. The optimal response functions $q_{i}\left(p, v_{i}\right), i=1,2, \ldots, n$, are continuous piece-wise linear nondecreasing functions of structure similar to that in Example 2. More precisely, if $\mathbf{0}<\xi_{1}<\xi_{2}<\cdots<\xi_{m}$ are the points of non-differentiability of the cost function $\boldsymbol{f}_{i}\left(\boldsymbol{q}_{i}\right)$, then 


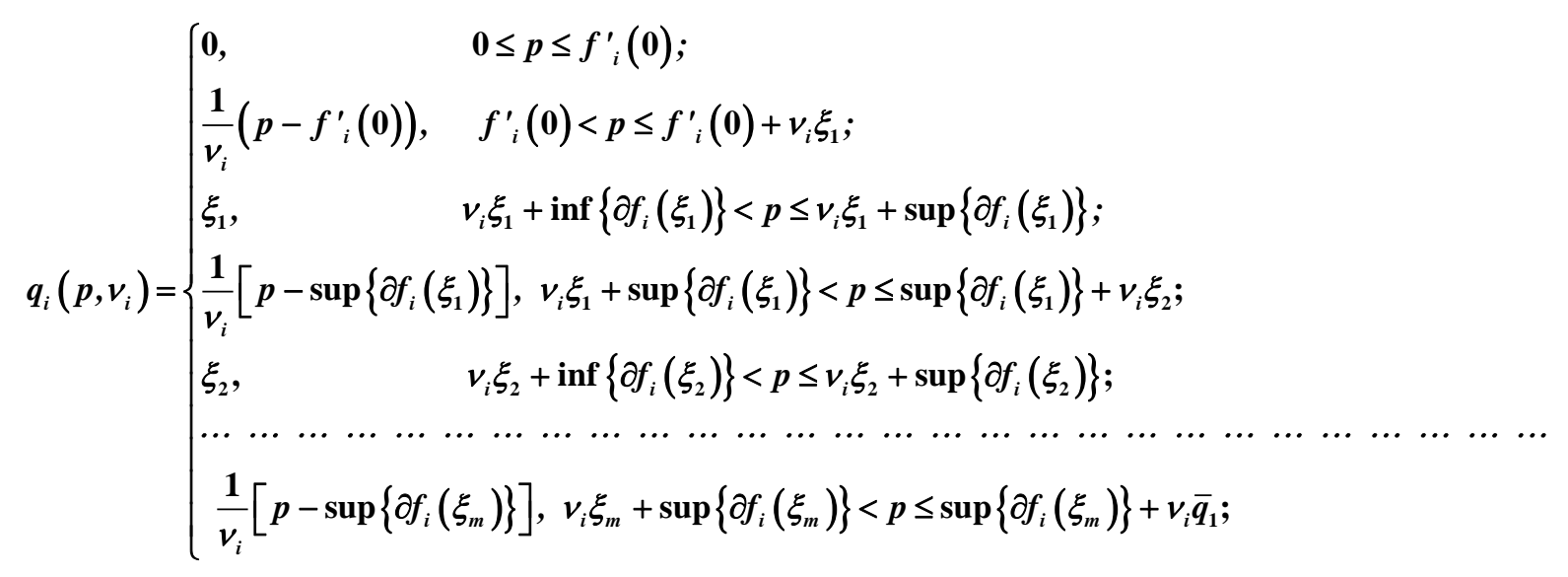

The latter construction is well-defined, as it is shown while proving Lemma 1 that

$$
\sup \left\{\partial f_{i}\left(\xi_{k-1}\right)\right\}=\inf \left\{\partial f_{i}\left(\xi_{k}\right)\right\}, \quad k=2,3, \ldots, m
$$

Now we introduce a notion of equilibrium which we call exterior (with parameters $\boldsymbol{v}_{\boldsymbol{i}}$ given exogenously).

Definition 1. The set $\left(p, q_{1}, \ldots, q_{n}\right)$ is called an exterior equilibrium for given influence coefficients $\left(\boldsymbol{v}_{1}, \ldots, v_{n}\right)$, if the market is balanced, i.e. condition (1) is satisfied, and for each $\boldsymbol{i}$ the maximum conditions (9) are valid.

To examine the existence of an equilibrium, we introduce some standard assumptions.

A1. The demand function is defined for prices $\boldsymbol{p} \in(\mathbf{0},+\infty)$ being a non-increasing and piecewise continuously differentiable one. At each of the finite number of discontinuity points there exist left- and right-hand limits of both the function itself (due to its monotonicity) and its derivative.

In what follows we are going to consider only the case when the participants set is constant independently from the values $\boldsymbol{v}_{\boldsymbol{i}}$ of the influence coefficients. To provide for that we make the following assumption, similar to that in [7] and [9].

A2. At the price $\boldsymbol{p}_{\mathbf{0}}=\max _{1 \leq i \leq n}\left\{\boldsymbol{f}^{\prime}{ }_{i}(\mathbf{0})\right\}$ for any $\boldsymbol{i}$ there exists an output volume $\boldsymbol{q}_{i}^{\mathbf{0}}$ such that $\boldsymbol{p}_{\mathbf{0}}=\inf \left\{\partial \boldsymbol{f}_{i}\left(\boldsymbol{q}_{i}^{0}\right)\right\}$ and moreover, $\sum_{i=1}^{n} q_{i}^{0}<g\left(p_{0}\right)$.

The latter condition guarantees that for any $\boldsymbol{v}_{\boldsymbol{i}}$ conditions (1) and (9) can hold together only for $\boldsymbol{p}>\boldsymbol{p}_{0} \geq \boldsymbol{f}^{\prime}{ }_{i}(\mathbf{0})$, and hence only for all $\boldsymbol{q}_{i}$ being strictly positive.

Now we introduce the upper limits for the total production of electricity by each firm. Indeed, let

$$
\bar{q}_{i}=\max \left\{\sum_{j=1}^{n_{j}} x_{i j} \mid \text { subject to (2)--(4) }\right\}, \quad i=1,2, \ldots n .
$$


For an exterior equilibrium to exist, it is crucial that the active demand $\boldsymbol{D}$ satisfy the following assumption.

A3. There exists a price value $\boldsymbol{p}^{1}$ such that

$$
G\left(p^{1}\right)+D<\sum_{i=1}^{n} \bar{q}_{i}(1-L O S S)
$$

Assumption A3 provides for existing the production power enough to satisfy the cumulative demand, given the active demand $\boldsymbol{D}$. Now, similar to [7] and [9], we can establish the following result.

Theorem 1. Under assumptions $\mathrm{A} 1$ and $\mathrm{A} 2$, for any $\boldsymbol{D} \geq \mathbf{0}$ satisfying $\mathrm{A} 3$, and $\boldsymbol{v}_{\boldsymbol{i}} \geq \mathbf{0}, \boldsymbol{i}=\mathbf{1}, \ldots, \boldsymbol{n}$, there exists an exterior equilibrium $\left(\boldsymbol{p}, \boldsymbol{q}_{1}, \ldots, \boldsymbol{q}_{n}\right)$. If the demand function is continuous and strictly decreasing, i.e. $\boldsymbol{g}(\boldsymbol{p}) \equiv \boldsymbol{G}(\boldsymbol{p})$ and $\boldsymbol{G}\left(\boldsymbol{p}_{1}\right)<\boldsymbol{G}\left(\boldsymbol{p}_{2}\right)$ for $\boldsymbol{p}_{\mathbf{1}}>\boldsymbol{p}_{\mathbf{2}}$, then the exterior equilibrium $\left(\boldsymbol{p}, \boldsymbol{q}_{1}, \ldots, \boldsymbol{q}_{n}\right)$ exists uniquely and depends continuously upon the parameters set $\left(D, v_{1}, \ldots, v_{n}\right)$.

Proof. Let us introduce the total optimal response of the producers to price $\boldsymbol{p}$ as follows

$$
Q(p)=\sum_{i=1}^{n} q_{i}\left(p, v_{i}\right)
$$

Due to Lemmas 1 and 2, the function $\boldsymbol{Q}(\boldsymbol{p})$ is piece-wise linear continuous non-decreasing function. Now introduce

$$
p_{*}=\sup \{p \mid Q(p)(1-L O S S) \leq G(p)+D\} .
$$

Assumptions A2 and A3 guarantee that this value exists and is finite. As $\boldsymbol{q}_{i}\left(\boldsymbol{p}, \boldsymbol{v}_{\boldsymbol{i}}\right)$ are continuous by Lemma 2 , and $\boldsymbol{G}(\boldsymbol{p})$ is left-continuous by assumption A1, then $\boldsymbol{Q}\left(\boldsymbol{p}_{*}\right)(\mathbf{1}-\boldsymbol{L O S S}) \leq \boldsymbol{G}\left(\boldsymbol{p}_{*}\right)+\boldsymbol{D}$. As the left limit of the demand function, $g(p)$, is right-continuous by assumption $A 1$, then were the inequality $\boldsymbol{Q}\left(\boldsymbol{p}_{*}\right)(\mathbf{1}-\mathbf{L O S S})<\boldsymbol{g}\left(\boldsymbol{p}_{*}\right)+\boldsymbol{D}$ valid, that would imply for some $\boldsymbol{p}>\boldsymbol{p}_{*}$ that

$$
Q\left(p_{*}\right)(1-L O S S)<g\left(p_{*}\right)+D \leq G(p)+D,
$$

which contradicts (13). Therefore, we have

$$
g\left(p_{*}\right)+D \leq Q\left(p_{*}\right)(1-L O S S) \leq G\left(p_{*}\right)+D,
$$

that is, the vector $\left(\boldsymbol{p}_{*}, \boldsymbol{q}_{1}^{*}, \ldots, \boldsymbol{q}_{n}^{*}\right)$ with $\boldsymbol{q}_{i}^{*}=\boldsymbol{q}_{i}\left(\boldsymbol{p}_{*}\right), \boldsymbol{i}=\mathbf{1}, \ldots, \boldsymbol{n}$, satisfies both (1) and (9), hence it is an exterior equilibrium.

The uniqueness of the exterior equilibrium in the particular case of strict monotonicity of the continuous demand function $g(p) \equiv \boldsymbol{G}(p)$ is evident, as the equilibrium is then determined by a unique intersection point of the graphs of the strictly decreasing continuous function $G(p)+D$ and the non-decreasing continuous function $\boldsymbol{Q}(\boldsymbol{p})(\mathbf{1}-\boldsymbol{L O S S})$. Thus, the equilibrium price $\boldsymbol{p}_{*}$, as well as the equilibrium production volumes $\boldsymbol{q}_{i}^{*}=\boldsymbol{q}_{i}\left(\boldsymbol{p}_{*}\right), \boldsymbol{i}=\mathbf{1}, \ldots, \boldsymbol{n}$, are determined uniquely, and the proof is completed. 


\section{Interior Equilibrium}

In order to introduce an interior equilibrium, we have to be able to realize the procedure of verification of the influence coefficients $v_{i}$ as it was given in [6]--[7],[9]. However, we cannot apply this procedure to our electricity model as the cost functions are piecewise linear ( $c f$. Lemma 1 and Example 1) and hence their second derivative either is zero or do not exist (at the junction points). To be able to verify the influence coefficients, we use a least squares approximation of the piecewise linear cost function $f_{i}$ by a quadratic cost function $\varphi_{i}$, $i=1,2, \ldots, n$, as follows.

For a fixed index $i$, let the cost function $f_{i}$ have the junction points $0=t_{0}<t_{1}<t_{2}<\ldots<t_{N}$. We determine the quadratic function $\boldsymbol{\varphi}_{i}\left(\boldsymbol{q}_{i}\right) \equiv \frac{1}{2} \boldsymbol{a}_{i} \boldsymbol{q}_{i}^{2}+\boldsymbol{b}_{i} \boldsymbol{q}_{i}+\boldsymbol{c}_{i}$ such that

$$
F\left(a_{i}, b_{i}, c_{i}\right) \equiv \sum_{k=0}^{N}\left[\frac{1}{2} a_{i} t_{k}^{2}+b_{i} t_{k}+c_{i}-f_{i}\left(t_{k}\right)\right]^{2}=\min _{a, b, c} \sum_{k=0}^{N}\left[\frac{1}{2} a t_{k}^{2}+b t_{k}+c-f_{i}\left(t_{k}\right)\right]^{2}
$$

Problem (14) always has a solution.

Example 3. To illustrate that, consider again the piecewise linear function from Example 1 and apply the least square method. The function in question has four junction points: $t_{\mathbf{0}}=\mathbf{0}<\boldsymbol{t}_{\mathbf{1}}=\mathbf{1 4}<\boldsymbol{t}_{\mathbf{2}}=\mathbf{1 6 . 1}<\boldsymbol{t}_{\mathbf{3}}=\mathbf{1 6 . 9 1 7}$ with $f\left(t_{0}\right)=0, f\left(t_{1}\right)=14, f\left(t_{2}\right)=18.2, f\left(t_{3}\right)=23.919$. By solving problem (14) we find the quadratic approximation cost function $\varphi(q) \equiv \frac{1}{2} a q^{2}+b q+c$ with $a=0.0890, b=0.5536, c=-0.7030$. The approximation error is $[\boldsymbol{F}(\boldsymbol{a}, \boldsymbol{b}, \boldsymbol{c})]^{1 / 2}=\mathbf{3 . 4 8}$. The second derivative is calculated as $\varphi^{\prime \prime} \equiv \boldsymbol{a}=\mathbf{0 . 0 8 9 0}$.

Now we can introduce the interior equilibrium. Assume that we have an exterior equilibrium $\left(\boldsymbol{p}, \boldsymbol{q}_{1}, \ldots, \boldsymbol{q}_{\boldsymbol{n}}\right)$ for the electricity market model with the piecewise linear cost functions $f_{i}$ replaced by the corrsponding quadratic approximations $\boldsymbol{\varphi}_{i}, \boldsymbol{i}=\mathbf{1}, \ldots, \boldsymbol{n}$. Assume also that this exterior equilibrium has occurred for some $\boldsymbol{v}_{\mathbf{1}}, \ldots, \boldsymbol{v}_{\boldsymbol{n}}$ and $\boldsymbol{D}$. One of the producers, say $\boldsymbol{k}$, temporarily changes his behavior by abstaining from maximization of the conjectured profit and making small fluxions around his equilibrium output volume $\boldsymbol{q}_{\boldsymbol{k}}$. In mathematical terms it is tantamount to restricting the model agents to the subset $\boldsymbol{i} \neq \boldsymbol{k}$ with the volume $\boldsymbol{q}_{\boldsymbol{k}}$ subtracted from the active demand. Variation of agent $\boldsymbol{k}$ production output is then reduced to the same absolute value variation (but to the opposite side) of the active demand $\boldsymbol{D}_{\boldsymbol{k}}=\boldsymbol{D}-\boldsymbol{q}_{\boldsymbol{k}}$. If we consider these variations being infinitesimal, we assume that by observing the corresponding variations of the equilibrium price, agent $\boldsymbol{k}$ gets the left-side and right-side derivatives of the equilibrium price with respect to the active demand, i.e. his left-side and right-side influence coefficients.

If the equilibrium point $\left(p, G=\sum_{i=1}^{n} q_{i}\right)$ belongs to the smooth part of the graph $L$ of the demand function, then these derivatives values coincide, and the consistent (verified) influence coefficient also must be equal to this value. However, if $(\boldsymbol{p}, \boldsymbol{G})$ is a corner point, then the coefficient $\boldsymbol{v}_{\boldsymbol{k}}$ is admitted as consistent if it lies between the left-side and right-side derivative values of the equilibrium price. Applying Theorem 1 [9] or, which is the same, formula (3) in [9], to calculate the derivatives, one has to remember that agent $\boldsymbol{k}$ is temporarily outside the equilibrium model, hence one has to exclude the addendum with the number $i=k$. Having that in mind, we come to the following criterion.

Consistency Criterion. At an exterior equilibrium $\left(\boldsymbol{p}, \boldsymbol{q}_{1}, \ldots, \boldsymbol{q}_{\boldsymbol{n}}\right)$ the influence coefficient $\boldsymbol{v}_{\boldsymbol{k}}$ is admitted as consistent if there exists a value $\boldsymbol{r}_{\boldsymbol{k}}$ such that

$$
\min \left\{G^{\prime}(p-0), G^{\prime}(p+0)\right\} \leq r_{k} \leq \max \left\{G^{\prime}(p-0), G^{\prime}(p+0)\right\}
$$


and

$$
v_{k}=\left(\sum_{i \neq k}\left(v_{i}+a_{i}\right)^{-1}-r_{k}\right)^{-1}
$$

where $\boldsymbol{a}_{i}=\boldsymbol{\varphi}_{i}{ }^{\prime \prime}, \boldsymbol{i}=\mathbf{1}, \ldots, \boldsymbol{n}, \boldsymbol{i} \neq \boldsymbol{k}$. The previous equality can be also rewritten as follows:

$v_{k}=\frac{1}{\sum_{i \neq k} \frac{1}{v_{i}+a_{i}}-r_{k}}$

Here the values $G^{\prime}(p-0)$ and $G^{\prime}(p+0)$ are taken at the point $\left(p, G=\sum_{i=1}^{n} q_{i}-D\right)$ of the demand function graph L. If $\boldsymbol{r}_{\boldsymbol{k}}=-\infty$ then $\boldsymbol{v}_{\boldsymbol{k}}=\mathbf{0}$.

Before we define the interior equilibrium we notice that when the equilibrium corresponds to a smooth point on the demand function graph then the numbers $\boldsymbol{r}_{\boldsymbol{k}}$ must be equal for all $\boldsymbol{k}$. Only if it is a vertex, i.e. if $\boldsymbol{G}^{\prime}(\boldsymbol{p}-\mathbf{0}) \neq \boldsymbol{G}^{\prime}(\boldsymbol{p}+\mathbf{0})$, the consistency criterion allows different values of $\boldsymbol{r}_{\boldsymbol{k}}$. Such a fact could be considered as natural if the graph's breakpoints are admitted as being exact reflections of the modeled situation. However, if one treats the breakpoint as a convenient idealization of smooth curves with rapidly changing slops, one has to demand the equality of all $\boldsymbol{r}_{\boldsymbol{k}}$ as it were the case with the smooth approximation of the demand curve. That is why such a case is considered distinctly in the definition of the interior equilibrium below, as it was done in [7] and [9].

Definition 2. The collection $\left(p, q_{1}, \ldots, q_{n}, v_{1}, \ldots, v_{n}\right)$ where $v_{k} \geq \mathbf{0 , i}=\mathbf{1}, \ldots, n$, is referred to as anterior equilibrium if, for the considered influence coefficients the collection $\left(\boldsymbol{p}, \boldsymbol{q}_{1}, \ldots, \boldsymbol{q}_{\boldsymbol{n}}\right)$ is an exterior equilibrium, and the consistency criterion is satisfied for all $\boldsymbol{k}$. Furthermore, if all $\boldsymbol{r}_{\boldsymbol{k}}$ in (4) have the same value, then the interior equilibrium is called strong.

The following theorem is an extension of Theorem 2 in [7], [9].

Theorem 2. Under assumptions $\mathbf{A 1}, \mathbf{A 2}$ and $\mathbf{A 3}$, there exists a strong interior equilibrium.

\section{Demand Structure and Equilibrium}

This section examines how variations of the demand structure affect the equilibrium price and the aggregate electricity output. We are mostly interested not in quantitative variations but the qualitative ones predicted by the above introduced model. Although Theorems 1 and 2 of the existence of equilibrium are valid in the more general case of piecewise quadratic cost functions, to understand the analysis better, we will first consider pure quadratic cost functions, as in [7].

Hence, recall again that we use the approximating quadratic cost functions of the form $\boldsymbol{\varphi}_{i}\left(\boldsymbol{q}_{i}\right)=\boldsymbol{a}_{i} \boldsymbol{q}_{i}^{2} / \mathbf{2}+\boldsymbol{b}_{\boldsymbol{i}} \boldsymbol{q}_{i}+\boldsymbol{c}_{\boldsymbol{i}}$ where $\boldsymbol{b}_{i}>\mathbf{0}$ and $\boldsymbol{a}_{\boldsymbol{i}}>\mathbf{0 , i}=\mathbf{1}, \ldots, \boldsymbol{n}$. If one is only interested in the strong interior equilibrium then formula (15) from the consistency criterion can be rewritten as follows:

$$
v_{k}=\frac{1}{\sum_{i \neq k} \frac{1}{v_{i}+a_{i}}-r}, \quad k=1, \ldots, n
$$


where $r \in[-\infty, \mathbf{0}]$. If $\boldsymbol{r}=-\infty$, then equation (16) has the unique solution $\boldsymbol{v}_{\boldsymbol{k}}=\mathbf{0}, \boldsymbol{i}=\mathbf{1}, \ldots, \boldsymbol{n}$. Otherwise, the following assertion is valid.

Lemma 3. ([7]). For any $\boldsymbol{r} \in \mathrm{J}_{-\infty, 0]}$ there is a unique solution of equation (16) $\boldsymbol{v}_{\boldsymbol{k}}=\boldsymbol{v}_{\boldsymbol{k}}(\boldsymbol{r}), \boldsymbol{k}=\mathbf{1}, \ldots, \boldsymbol{n}$, that depend continuously upon $\boldsymbol{r}$. Furthermore, $\boldsymbol{v}_{\boldsymbol{k}}(\boldsymbol{r}) \rightarrow \mathbf{0}$ when $\boldsymbol{r} \rightarrow-\infty$, and $\boldsymbol{v}_{\boldsymbol{k}}(\boldsymbol{r})$ strictly increases up to $\boldsymbol{v}_{\boldsymbol{k}}(\mathbf{0})$ as $\boldsymbol{r}$ increases up to zero.

Relations (9) for the approximating quadratic functions give the linear dependences $q_{i}\left(p, v_{i}\right)=\frac{\boldsymbol{p}-\boldsymbol{b}_{\boldsymbol{i}}}{\boldsymbol{v}_{\boldsymbol{i}}+\boldsymbol{a}_{\boldsymbol{i}}}$. Thus for each $r \in[-\infty, 0]$ one can determine $\boldsymbol{v}_{\boldsymbol{k}}, \boldsymbol{i}=\mathbf{1}, \ldots, \boldsymbol{n}$ from (16) and then construct the aggregate output function

$$
Q(p, r)=\sum_{i=1}^{n} \frac{p-b_{i}}{v_{i}+a_{i}}
$$

It is immediate that $Q(p, r)$ strictly increases by $\boldsymbol{p}$ and strictly decreases with respect to $\boldsymbol{r}$. Therefore the intersection points of the straight lines $\boldsymbol{G}=\boldsymbol{Q}(\boldsymbol{p}, \boldsymbol{r})$ with the demand curve move steadily to the right as $\boldsymbol{r}$ grows from $-\infty$ up to $\mathbf{0}$. The straight line $\boldsymbol{G}=\boldsymbol{Q}(\boldsymbol{p},-\infty)$ describes the dependence of the aggregate output upon the price $\boldsymbol{p}$ in case of the perfect competition, when the producers exclude the possibility of their individual influence on the price. On the other hand, the straight line $G=Q(p, 0)$ corresponds to the maximum (for the given cost functions) claims of the individual influence.

First consider the situation of Fig. 1. Here the passive demand $\boldsymbol{G}(\boldsymbol{p})$ is zero if $\boldsymbol{p}>\boldsymbol{p}_{*}$ but equals $\boldsymbol{G}_{\mathbf{0}}$ when $\boldsymbol{p} \leq \boldsymbol{p}_{*}$, that is, there is a momentary leap of demand. The right-side limit $\boldsymbol{g}(\boldsymbol{p})$ here is zero if $\boldsymbol{p} \geq \boldsymbol{p}_{*}$ and equals $\boldsymbol{G}_{\mathbf{0}}$ when $\boldsymbol{p}<\boldsymbol{p}_{*}$. A positive active demand $\boldsymbol{D}_{\mathbf{0}}$ somewhat lifts the demand curve up. The straight lines numbered $\mathbf{0}$ to $\mathbf{2}$ are the graphs of the aggregate production for various values of $\boldsymbol{r}$. More precisely, number $\mathbf{0}$ is attached to the case $\boldsymbol{r}=-\infty$, i.e. the perfect competition. Number $\mathbf{1}$ is assigned to the case $\boldsymbol{r}=\mathbf{0}$, whereas $\mathbf{2}$ is numbering the case of an intermediate value $\overline{\boldsymbol{r}}$ in which the aggregate production graph comprises the point $\left(p_{*}, D_{0}+G_{0}\right)$. Because $G^{\prime}\left(p_{*}-\mathbf{0}\right)=\mathbf{0}$ and $G^{\prime}\left(p_{*}+\mathbf{0}\right)=-\infty$ at that point, then it represents an interior equilibrium. Another interior equilibrium is associated with the graph point $\left(\boldsymbol{p}_{\mathbf{1}}, \boldsymbol{D}_{\mathbf{0}}\right)$ where $G^{\prime}\left(p_{1}-0\right)=G^{\prime}\left(p_{1}+\mathbf{0}\right)=\mathbf{0}$ and $r=0$. Strictly speaking there is the third equilibrium in this situation, namely the point $\left(\boldsymbol{p}_{*}, \boldsymbol{D}_{\mathbf{0}}\right)$ which corresponds to some $\boldsymbol{r} \in(\overline{\boldsymbol{r}}, \mathbf{0})$, as in this point one has $\boldsymbol{G}^{\prime}(\boldsymbol{p}-\mathbf{0})=-\infty$ and $\boldsymbol{G}^{\prime}(\boldsymbol{p}+\mathbf{0})=\mathbf{0}$. However, the latter equilibrium is unstable: any growth of the active demand makes the price jump up to the value $p_{1}$.

Now let the electricity market have the state $\left(\boldsymbol{p}_{*}, \boldsymbol{D}_{\mathbf{0}}+\boldsymbol{G}_{\mathbf{0}}\right)$ and the active demand $\boldsymbol{D}$ starts growing up thus lifting up the demand curve. Until $\boldsymbol{D} \leq \boldsymbol{D}_{\mathbf{0}}+\boldsymbol{\delta}$ the situation keeps changing continuously with the price $\boldsymbol{p}_{*}$ kept intact and the demand $\boldsymbol{D}+\boldsymbol{G}_{\mathbf{0}}$ satisfied completely. The production of electricity increases on account of diminishing of the equilibrium influence coefficients (i.e. on account of $\boldsymbol{r}$ decreasing). When the breakpoint of the demand curve reaches line $\mathbf{0}$ (that corresponds to $\boldsymbol{D}=\boldsymbol{D}_{\mathbf{0}}+\boldsymbol{\delta}$ and to the perfect competition regime) then the price cannot be supported unchanged as $\boldsymbol{v}_{\boldsymbol{i}}=\mathbf{0}, \boldsymbol{r}=-\infty$, and they cannot be lowered anymore.

The next stage of the process is reflected in Fig. 2. If the active demand belongs to the interval $\left(D_{0}+\delta, D_{0}+G_{0}+\delta\right)$, then the equilibrium is represented by the point $\left(p_{*}, D_{0}+G_{0}+\delta\right)$ which corresponds to the perfect competition regime. Here neither the electricity price nor the electricity output is affected by the active 
demand value, but a deficit appears because the aggregate demand $\boldsymbol{D}+\boldsymbol{G}_{\mathbf{0}}$ is not satisfied. Along with the active demand growing up to the value $\boldsymbol{D}_{\mathbf{0}}+\boldsymbol{G}_{\mathbf{0}}+\boldsymbol{\delta}$, the deficit will also go up to the passive demand value $\boldsymbol{G}_{\mathbf{0}}$.

The third stage comes with the active demand exceeding the value of $\boldsymbol{D}_{\mathbf{0}}+\boldsymbol{G}_{\mathbf{0}}+\boldsymbol{\delta}$. At this moment the equilibrium point $\left(\boldsymbol{p}_{*}, \boldsymbol{D}_{\mathbf{0}}+\boldsymbol{G}_{\mathbf{0}}+\boldsymbol{\delta}\right)$ loses stability, the price jumps to the value $\boldsymbol{p}^{*}>\boldsymbol{p}_{*}$ and the deficit vanishes due to the elimination of the passive demand. The electricity market jumps to a qualitatively new state. Although the aggregate production output has not fallen down, the prices are established on a level higher than the marginal costs. Further growing active demand will be satisfied completely on the account of the corresponding growth of price.

Now suppose that the active demand starts going down. It is evident that the process will not be reversal one. The equilibrium point will stay on line $\mathbf{1}$ until the active demand reaches the value $\boldsymbol{D}_{\mathbf{1}}<\boldsymbol{D}_{\mathbf{0}}$ and the price becomes equal to $\boldsymbol{p}_{*}$ but now at the lower production level than in the beginning of the process. Further falling active demand will force the electricity market to jump to the initial state: the price $\boldsymbol{p}_{*}$ being intact, the output volume will increase up to $\boldsymbol{G}_{\mathbf{0}}+\boldsymbol{D}_{\mathbf{1}}$ having satisfied both the active and passive demands

completely. However, if the active demand returns only to its initial state $\boldsymbol{D}_{\mathbf{0}}$, the electricity market keeps staying at the equilibrium point $\left(\boldsymbol{p}_{1}, \boldsymbol{D}_{\mathbf{0}}\right)$ of Fig. 1, the price will be fixed higher the critical price $\boldsymbol{p}_{*}$ for the passive demand, and only the active demand $\boldsymbol{D}_{\mathbf{0}}$ will be satisfied.

If the demand curve has not single but several steps, the behavior of prices will be similar. The only difference is in that the process will be repeated as many times as many steps there are to the right off line $\mathbf{0}$ in Fig. 1. Each leap of prices corresponds to a switch to a new regime, with an appropriate group of consumers abandoning the market and thus eliminating the deficit that had arisen before that.

Now consider the case when the active demand is stable but the passive demand structure changes on account of, say, a variation of the consumption ability of a part of consumers. We illustrate this process on an example of two groups of consumers in the absence of active demand. Again we restrict ourselves to an ideal case when the demand curve is a step function with two steps (Fig. 3). The price $p_{1}^{*}$ is critical for the poorer group of consumers with consumption volume $G_{1}$, whereas the price $p_{2}^{*}>p_{1}^{*}$ is such for the richer group with consumption volume $\boldsymbol{G}_{\mathbf{2}}$. The lines numbered by $\mathbf{0}$ and $\mathbf{1}$ have the same meaning as in the previous example, i.e. they are the aggregate production output graphs in the perfect competition regime and the maximum monopolistic tendencies regime, respectively. The intermediate graphs numbered as $\mathbf{2}$ and $\mathbf{3}$ are associated with two interior equilibria $\left(p_{1}^{*}, G_{1}+G_{2}\right)$ and $\left(p_{2}^{*}, G_{2}\right)$.

Now suppose that the consumption ability of the first (the poorer) group of consumers goes down. This process can be modeled by diminishing the critical price $p_{1}^{*}$. For simplicity purpose, we ignore a possible decrease of the consumption volume $\boldsymbol{G}_{\mathbf{1}}$. Again, the first stage of the considered process consists in lowering of the influence coefficients thus approaching the perfect competition regime.

This feature will be exposed by the market price going down with the production output unchanged and the aggregate demand satisfied. The price will be equal to the critical one for the poorer group of consumers. This will be taking place until the critical price reaches the level $p^{1}$ with the production switching to the perfect competition regime.

At the second stage when the critical price for the first group of consumers lies in the interval $\left(p_{0}^{1}, p^{1}\right)$, the market price keeps going down along with the critical one, the perfect competition keeps being valid, but the aggregate production volume starts decreasing and a deficit arises (see Fig. 4). The deficit will increase as the price 
approaches the value $\boldsymbol{p}_{\mathbf{0}}^{\mathbf{1}}$. If the consumption ability keeps lowering then the market price jumps up to the level $\boldsymbol{p}_{2}^{*}$, and the electricity market makes a leap to the new equilibrium regime $\left(\boldsymbol{p}_{2}^{*}, \boldsymbol{G}_{2}\right)$. The poorer group of consumers abandons the market completely with the deficit eliminated.

If the consumption ability of the first group recovers, the process is not reversed completely (if no special extraneous efforts are made).

\section{Conclusion}

The qualitative analysis of the equilibrium behavior in dependence on the active demand level for the electricity market model is realized based upon that performed in [7] and [9] for the case of pure quadratic ([7]) and piecewise quadratic ([9]) cost functions. The model in question necessarily has piecewise linear cost function. Such cost functions arise often when the model agents have several distinct production facilities among which they have to distribute the production volume so that the total cost be minimal. Therefore, to introduce the notion of interior equilibrium, we have to approximate these piecewise linear cost function by pure quadratic ones. For thus introduced pure quadratic approximating cost function, we are able to realize the qualitative analysis of the behavior of electricity prices and production outputs for the electricity market model.

Acknowledgement: This work was financially supported by the University of Nuevo Leon (UANL) Project PAICyT No. CA751-02.

\section{References}

1. Ruffin R.J. Cournot oligopoly and competitive behavior. Rev. Econ. Studies. 1971. V. 38(4). N. 116.

2. Sherali H.D., Soyster A.L. and Murphy F.H. Stackelberg -Nash-Cournot equilibria: characterizations and computations. Oper. Res. 1983. V. 31. N. 2.

3. Novshek W. On the existence of Cournot equilibrium. Rev. Econ. Studies. 1985. V. 52(1). N. 168.

1. 4. Bulavsky, V.A., Kalashnikov, V.V. One-Parametric Method to Study an Equilibrium. Economics and Mathematical methods. 1994. V. 30. N. 4. (In Russian)

4. Bulavsky, V.A., Kalashnikov, V.V. Equilibrium in Generalized Cournot and Stackelberg Models. Economics and Mathematical methods. 1995. V. 31. N. 3. (In Russian)

5. Bulavsky, V.A. An Imagined Experiment in the Framework of the Generalized Cournot Model. Economics and Mathematical methods. 1996. V. 32. N. 2. (In Russian)

6. Bulavsky, V.A. Structure of Demand and Oligopolistic Equilibria. (Private communication)

7. Quick, D.M., and Carey, J.M. Transmission Capacity and Market Power: the Effect on a Dominant Generation Firm. Energy Policy. 2002. V. 30.

8. Kalashnikova, N.I. Structure of Demand and Equilibrium in Oligopolistic Models with Piecewise Quadratic Cost Functions. Journal of Business and Economics Research (to appear). 


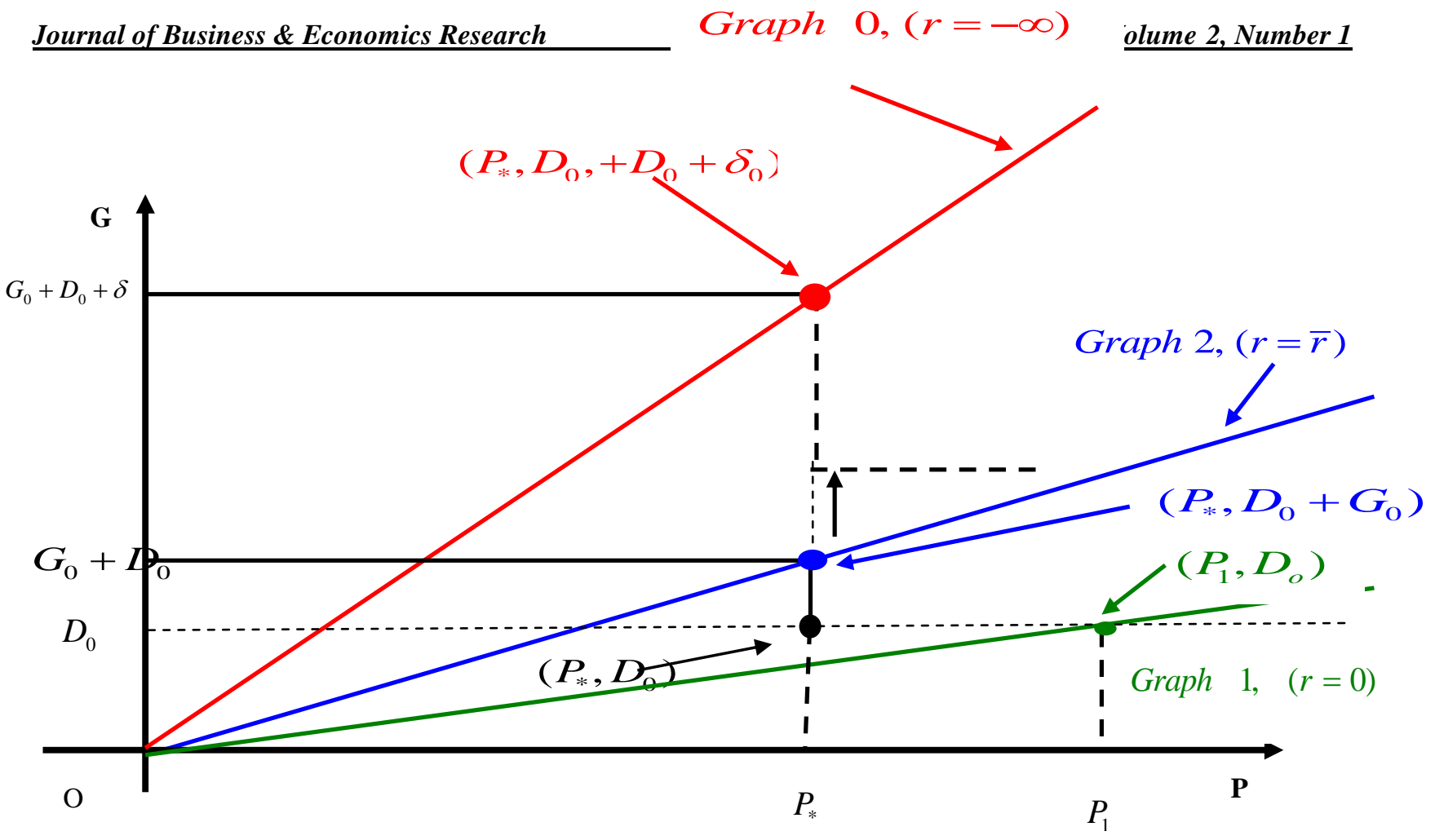

Figure 1

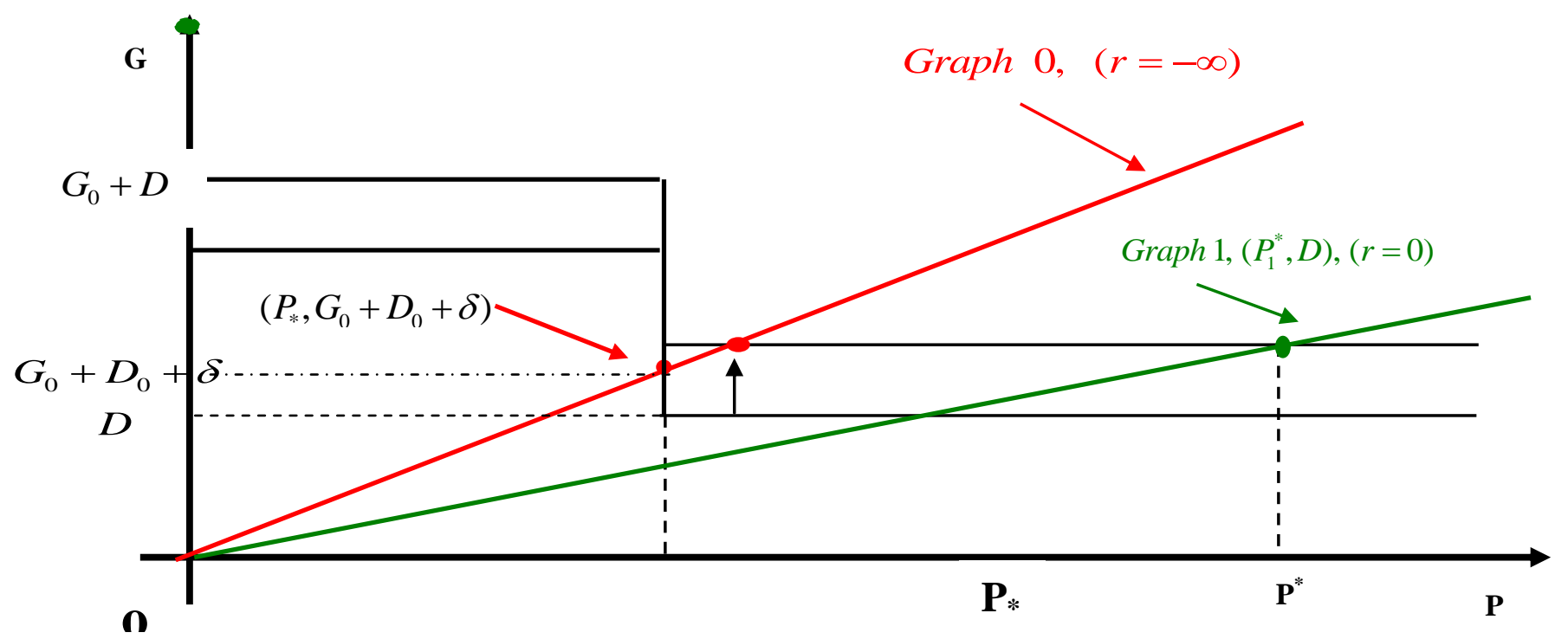

Figure: 2 


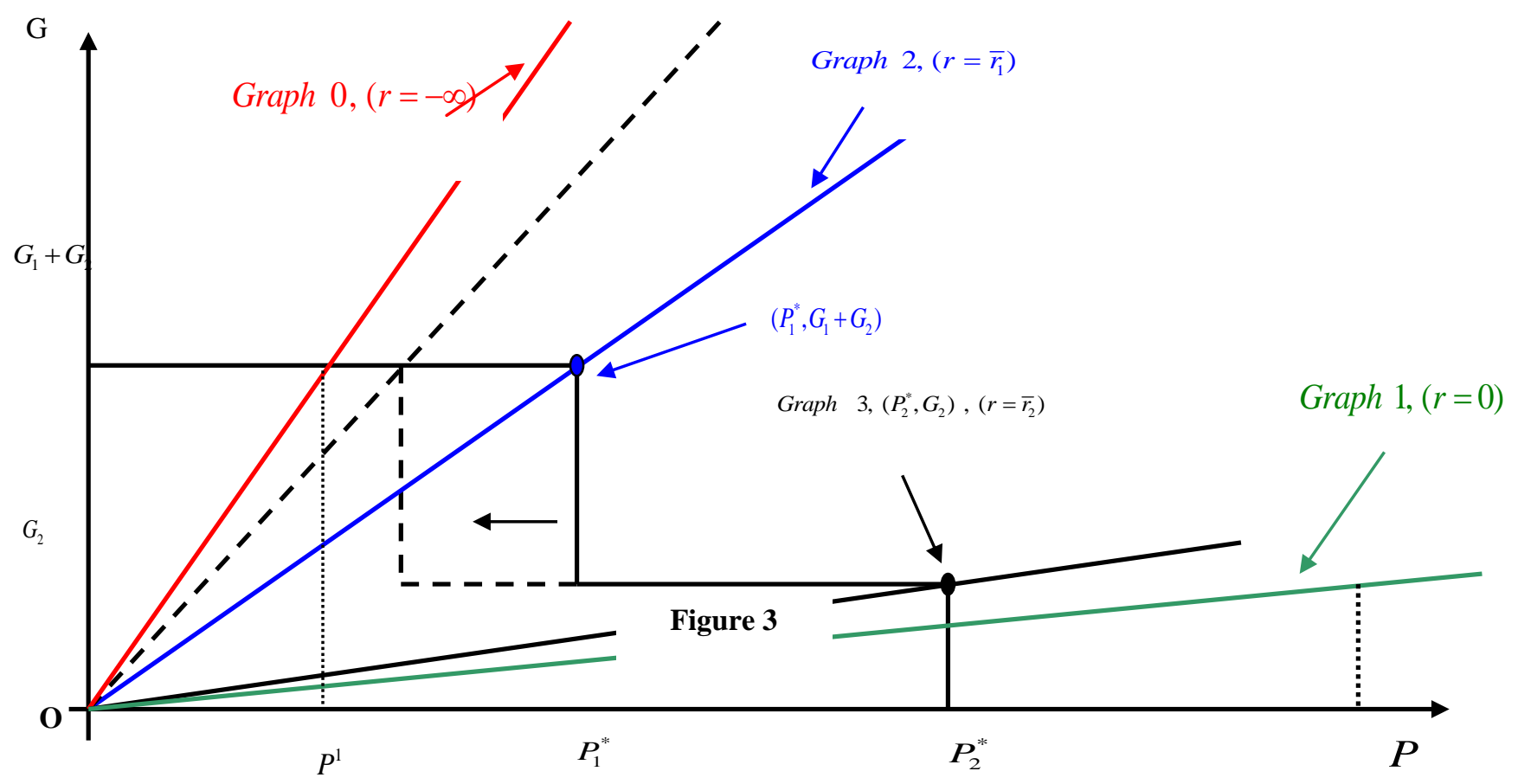

Figure 3

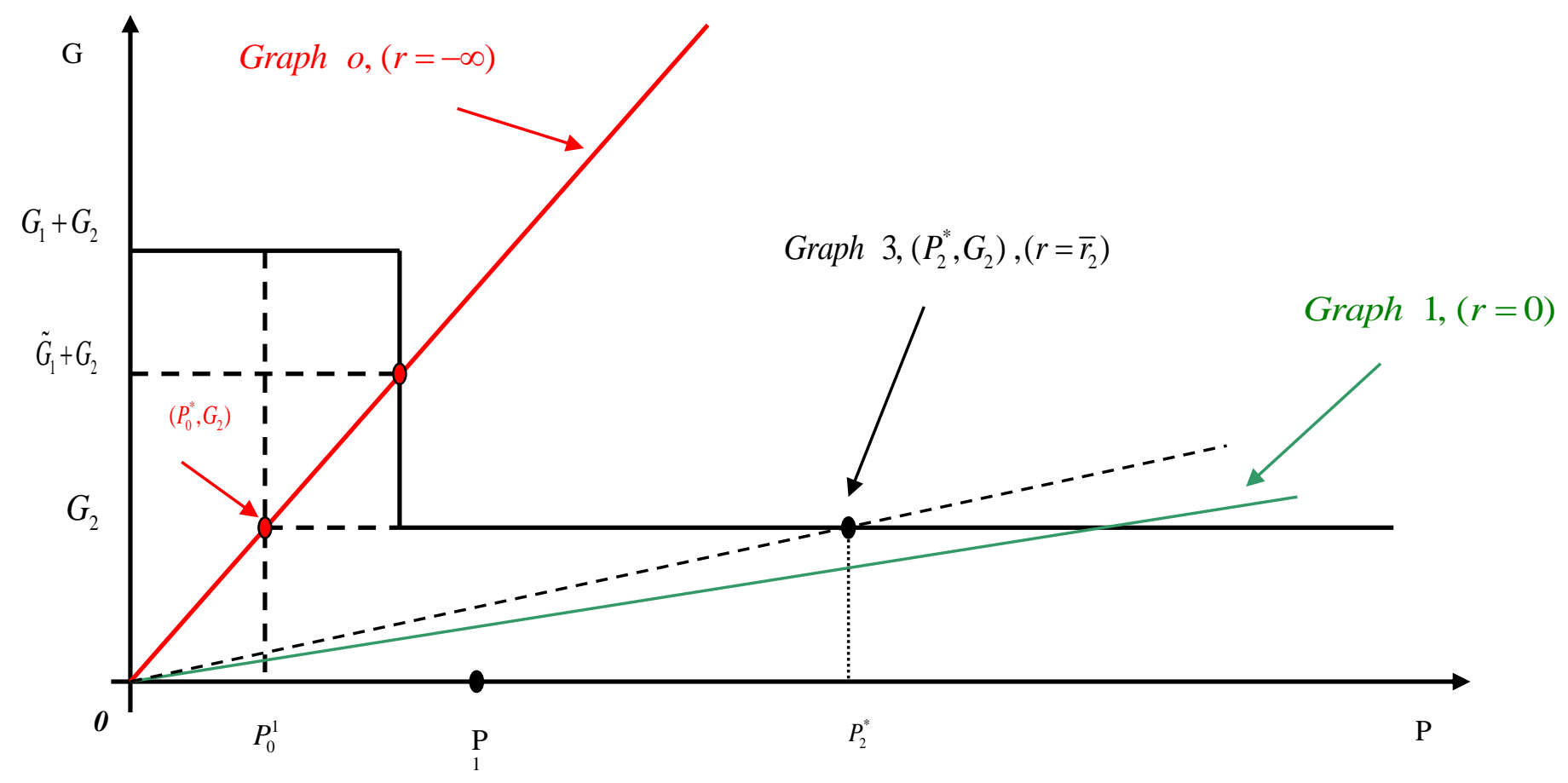

Figure 4 